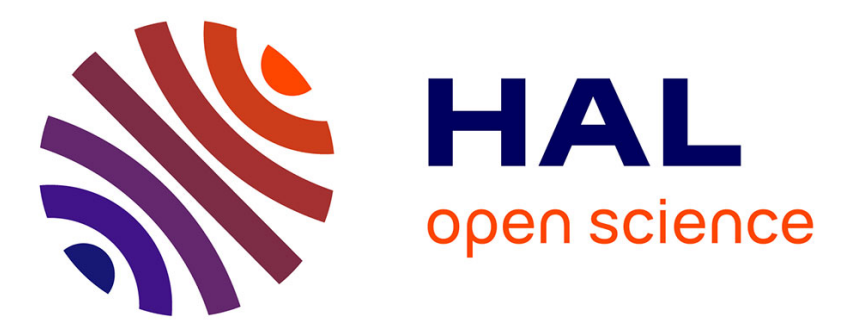

\title{
Integrating Imperfect Information in the Deterioration Modeling of Torrent Protection Measures for Maintenance and Reliability Assessment
}

Nour Chahrour, Jean-Marc Tacnet, Christophe Bérenguer

\section{- To cite this version:}

Nour Chahrour, Jean-Marc Tacnet, Christophe Bérenguer. Integrating Imperfect Information in the Deterioration Modeling of Torrent Protection Measures for Maintenance and Reliability Assessment. ESREL 2021 - 31st European Safety and Reliability Conference, Sep 2021, Angers, France. pp.26302637, 10.3850/978-981-18-2016-8_670-cd. hal-03450061

\section{HAL Id: hal-03450061 \\ https://hal.science/hal-03450061}

Submitted on 6 Dec 2021

HAL is a multi-disciplinary open access archive for the deposit and dissemination of scientific research documents, whether they are published or not. The documents may come from teaching and research institutions in France or abroad, or from public or private research centers.
L'archive ouverte pluridisciplinaire HAL, est destinée au dépôt et à la diffusion de documents scientifiques de niveau recherche, publiés ou non, émanant des établissements d'enseignement et de recherche français ou étrangers, des laboratoires publics ou privés.

\section{(1) (1) $\$$}

Distributed under a Creative Commons Attribution - NonCommercial - NoDerivatives $\mid 4.0$ 


\title{
Integrating Imperfect Information in the Deterioration Modeling of Torrent Protection Measures for Maintenance and Reliability Assessment
}

\author{
Nour Chahrour \\ Univ. Grenoble Alpes, INRAE, ETNA, 38000 Grenoble, France. \\ Univ. Grenoble Alpes, CNRS, Grenoble INP, GIPSA-lab, 38000 Grenoble, France. \\ E-mail: nour.chahrour@inrae.fr \\ Jean-Marc Tacnet \\ Univ. Grenoble Alpes, INRAE, ETNA, 38000 Grenoble, France. \\ E-mail: jean-marc.tacnet@inrae.fr \\ Chistophe Bérenguer \\ Univ. Grenoble Alpes, CNRS, Grenoble INP, GIPSA-lab, 38000 Grenoble, France. \\ E-mail: christophe.berenguer@grenoble-inp.fr
}

Among several types of natural risks' protection measures, check dams are the most dominant in French mountains. Over their lifetime and while being subjected to severe phenomena such as torrential floods, the check dams' efficacy will be affected and therefore the level of protection provided by these structures will be reduced. Available budgets oblige risk managers to establish priorities between different maintenance strategies to be applied on structures that require maintenance. Modeling the dynamic deterioration of check dams and making decisions on their maintenance always depend on the amount of available data, diverse sources of data, assumptions, etc. This paper focuses on assessing the influence of uncertain inputs on the outputs obtained from a check dam's deterioration and maintenance model. The end purpose of such analysis is to support check dams' maintenance decision-making under the effect of information imperfection.

Keywords: Natural risk, check dams, deterioration modeling, efficacy assessment, maintenance decision-making, information imperfection.

\section{Introduction}

Mountain territories share common particular topographical and geomorphological features that makes them remarkably exposed to natural hazards. Steep slopes, narrow streams, and short valleys in mountains boost gravitational forces thus triggering gravitational phenomena of various natures. These phenomena (e.g. torrential floods, debris flows, etc.) have similar physical behavior and are usually characterized in terms of magnitude (e.g. discharge rate), intensity (e.g. velocity), effect (e.g. erosion) and frequency (probability of occurrence). They all have the potential to induce severe damages and to put exposed elements at risk.

In the context of natural hazards, risk is defined as a combination of hazard (intensity and frequency) and potential damage (vulnerability of elements at risk). Generally, risk management involves identifying the risk level and then choosing the best strategy for prevention or mitigation. The preventive management of torrential risks is largely based on several types of structural protec- tion measures constructed in different zones of the watershed. Each type (e.g. check dams, retention dams, dykes) has its own function depending on the component of hazard it acts on. For example, torrent check dams are civil engineering structures, designed to prevent sediment production in the catchment basin. They are implemented in series in the flow channel aiming to prevent longitudinal and lateral erosion.

To be effective, these structures have to fulfill their functional requirements and to be stable from a mechanical point of view considering external and internal stability equilibrium justifications. Over time, these structures deteriorate due to aging and the direct (impact) and indirect (geomorphic activity, domino effect) consequences of the hazards. Consequently, their structural and functional efficacy are influenced according to their level of degradation. On a global risk management level, a key issue lies in assessing protection structures' efficacy over their lifetime. This allows risk managers (1) to identify the level of security received by the exposed protected issues (people, 
properties, infrastructures) and (2) to set priorities concerning inspection and maintenance interventions.

Since the 19th century, the French government has initiated a policy known as RTM standing for Restoration des Terrains en Montagne, for managing land use and protecting mountains. The main role of RTM is to provide assistance regarding mitigation actions against natural risks and to guarantee the efficacy of protection structures. However, specific available budgets are provided by the state, dedicated for natural risk prevention and management. Consequently, managing protection systems necessitates making decisions depending on the structure's technical efficacy (structural, functional) in addition to its socioeconomic efficacy in reducing risk taking into consideration the cost of possible interventions: inspection, preventive or corrective maintenance.

Ideal decisions are made when real data related to natural events and structures' behaviors are available. In the addressed context, these data are often missing or imperfect (incomplete, imprecise...). Available information as well, may be affected by the precision of the design data of the structures (e.g. dimensions, materials' properties, soil characteristics), events data (e.g. intensity, frequency), and also by the validity of the assumptions made based on experts' knowledge and skills. Risk management decisions are therefore influenced by the quality of the heterogeneous information coming from more or less reliable sources such historical data, expert assessments, and numerical simulations. Taking this imperfection into account through the diagnosis and decision-making process is therefore essential and challenging (Carladous et al., 2019).

In a recent research work, a multi-disciplinary approach is developed in order to support maintenance decision-making of a check dam subjected to torrential floods (Chahrour et al., 2021). It starts by a physics-based model that permits modeling the dynamic deterioration of the dam taking into account interactions between structural (external stability) and functional (scouring) failure modes. The integrated approach also provides a stochastic model of time-dependent state evolution of the dam using Petri net tools in order to assess maintenance policies considering economic aspects. The model has been applied on a real dam constructed in Manival torrent in the French alps. Most of the data used were extracted from ONF-RTM database. On one hand, some of these data may be imperfect due to inconsistency, imprecision, and uncertainties. On the other hand, the data provided is incomplete and thus all missing information are assumed and assessed by experts in the field. The reliability of results may be therefore influenced by this imperfection which was not yet analyzed in the existing approach. On this basis, final results should be assessed with regard to information imperfection.

This paper aims to show how different types of information imperfection propagate within a check dam's deterioration and maintenance model. It also highlights the parameters that greatly influence the final results involved in different stages of the integrated approach. This is achieved by using HYRISK, a R-package that enables propagating both aleatory and epistemic uncertainties and quantifying the sensitivity of the proposed model outputs to all possible uncertainties. The obtained results in turn reveal how protection structures' maintenance decisions can significantly be influenced by the quality of the used information.

The next section, describes the origin and the nature of information imperfection and briefly reviews some of the methods used for its propagation. Section 3 summarizes the check dam's deterioration and maintenance model developed by (Chahrour et al., 2021) and describes the hybrid approach used for assessing different forms of information imperfection involved within the model. In Section 4, HYRISK analysis is applied to the check dam's model in order to reveal some of the expected results. The application, results and discussion focuses on parameters used in the physics-based model involved within the overall decision-making process where imperfection in the data is significantly present. Conclusions and future perspectives are presented in Section 5.

\section{Multi-scale Decision-Making Process Under Information Imperfection}

In watersheds, natural risk management reflects a complex decision process that involves several decisions made depending on multi spatial (fig. 1) and temporal (before, during and after event) scales. Decisions often result from a combination of several expert assessments involved in hazard, vulnerability, and risk analysis contributing to various types of assessments. Indeed, final decisions often result by combining multi-scale assessments concerning the (i) phenomena characteristics, (ii) protection system's design and efficacy (iii) risk level imposed on downstream elements and (iv) available budgets. The final objective behind all decisions is to reduce the risk level imposed downstream and thus to provide sufficient protection to exposed elements.

Whatever the type of hazard, risk management decision process starts by data acquisition, numerical modelling for hazard and vulnerability assessment, and ends up by making decisions concerning mitigation measures. Apparently, all decisions are influenced by the quality of the used data, the credibility of sources (database, experts) from which these data were acquired and by the reasonability of adopted scenarios and assumptions (expertise). The fact that information imper- 


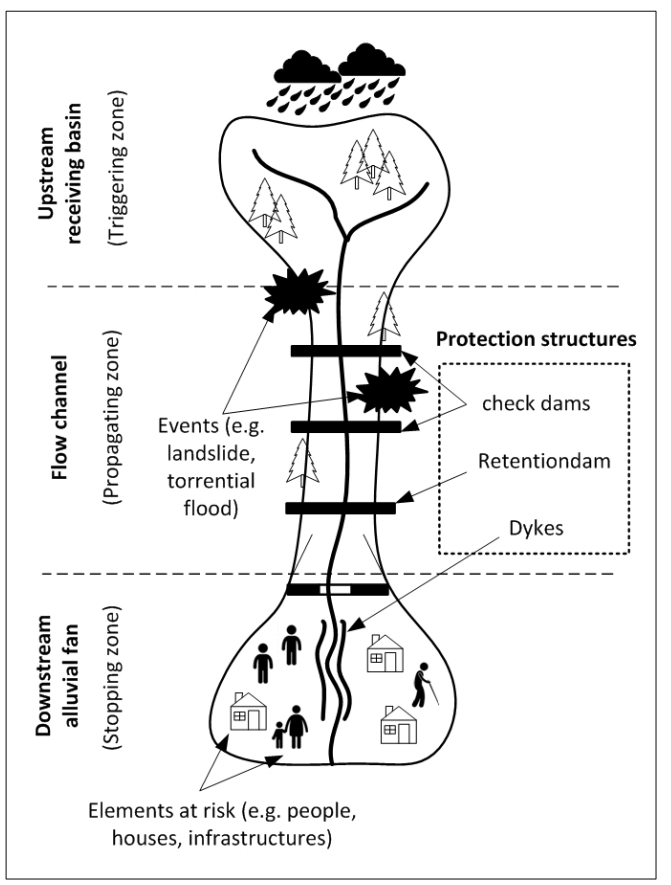

Fig. 1. Spatial partitioning of torrential watersheds.

fection propagates between the different stages of the process puts risk managers and stakeholders in a doubt whether the final adopted decisions are optimal or not.

\subsection{Types of imperfections relative to information quality}

Generally, information can be described according to several features and characteristics. The type, content and quality of information significantly influence any decision. Information can be either (i) subjective, based on personal opinions and points of view or (ii) objective, based on facts, measurements and observations. Obviously, subjective information are not always suitable for decisionmaking especially in critical fields related to risk and safety analysis, business, politics, etc. Its content can be either (i) quantitative, expressed by numerical values (e.g. physical quantities) or (ii) qualitative, categorized according to different references (e.g. low, medium, high). Information quality refers to imperfection which can be categorized into four different types:

(1) Inconsistency concerns the conflict between several sources of information. For example, two experts can provide different points of view).

(2) Imprecision relates to the insufficiency of information. For example, a range of values can correspond to a physical quantity, in which the correct value is bounded by a lower and an upper interval values.

(3) Incompleteness corresponds to the partial availability or total lack of information. For example, the volume of a specific debris flow event could be missing.

(4) Uncertainty is the relation between the real state and the assessment of a specific situation. Two different types of uncertainty can be distinguished: aleatory uncertainty resulting from a stochastic behaviour, and epistemic uncertainty resulting due to imprecision or lack of knowledge (Tacnet et al. 2014).

\subsection{Theoretical frameworks coping with information imperfection}

Natural risk assessment and management correspond to situations where there is low availability of data. In such cases, experts' judgements are required to compensate for this lack of data. Therefore, achieving wise decisions necessitates theoretical frameworks that enables representing and combining all types of information imperfection. Several theories already exist to cope with information imperfection propagation and decisionmaking processes. Those mainly adopted in natural risk context are briefly reviewed below.

Probability theory is a classical approach, widely used in natural hazard context to characterize and propagate aleatory uncertainty in the model by assuming probability distributions for uncertain variables estimated by experts.

Possibility theory (Dubois 1989) can represent both imprecise and uncertain (aleatory and epistemic) information using possibility distributions. It deals with intervals instead of single evaluations. Indeed, it better reflects the imprecision that prevails expert judgment in which the wider the interval is, the more confident the expert is.

Fuzzy sets theory (Zadeh 1965) deals with ambiguous information and imprecise evaluations. Fuzzy numbers allow incorporating uncertainty on parameters. They also relates ambiguous (e.g. linguistic) variables to numerical (quantitative) values.

Evidence theory (Shafer, 1976) (also called belief function theory) represents imprecision, uncertainty, inconsistency and incompleteness in the provided information. It also deals with information fusion in which it permits merging information acquired from conflicting sources.

In the context of risk analysis, the different types of imperfection are encountered. Separately, classical theories fail to cope with all aspects of imperfection. Therefore, it is essential to seek for other alternatives that consider the heterogeneous, ambiguous, imprecise and conflicting information 
actually accessible by risk managers, decisionmakers and stakeholders.

\subsection{Uncertainty propagation through hybrid approaches}

Probabilistic models and Monte-Carlo simulation are generally adopted for representing uncertainties and propagating them through the models. However, uncertainty analysts agreed on the fact that probabilistic approaches are not adequate for modeling epistemic uncertainties (Walley, 1991). Indeed, recent approaches differentiate between aleatory and epistemic uncertainty and model them by means of different theories but still within an integrated framework (Helton and Oberkampf, 2004). For example, in risk context, aleatory uncertainty is usually modeled by conventional probability distributions and epistemic uncertainty by possibility distributions or fuzzy calculus (Chojnacki et al., 2010).

Combining the theories of probabilities, possibilities, fuzzy sets and belief functions makes it possible to represent both variability (randomness) and imprecision, related to the imperfect knowledge provided by experts regarding different variables involved in the model. Such integrated frameworks, often referred to as 'hybrid' approaches, have been highly appreciated for addressing different aspects of information imperfection, especially in risk assessments.

\section{Integrated Model for Check Dam's Maintenance Decision-Making}

Torrent check dams are constructed in mountains to resist natural phenomena and thus to protect exposed issues. Nonetheless, they may be subjected to functional (scouring, lateral bypass) and/or structural (loss in stability, reduction in materials' strengths) failures while being subjected to high intensities phenomena. Consequently, their efficacy in reducing risk decreases over time. The optimization of maintenance strategies requires having a comprehensive knowledge regarding both, the technical (structural, functional) and the socioeconomic efficacy of the structure. Indeed, recent research works are focusing on developing innovative decision-support approaches that prioritize maintenance strategies while analyzing the timedependant efficacy reduction of protection structures in mountains.

Efficacy assessment requires inspecting structures at regular basis. Since failure modes associated to these structures are perfectly known (Tacnet and Richard 2010), state indicators corresponding to different types of failures are defined to help perform the assessment. The ideal situation for estimating these quantitative indicators consists in acquiring real data related to natural events (intensity, frequency), torrent's characteristics (topography, geotechnical data), protection structures' designs (dimensions, material properties). However, these data are often based on assumptions and expert judgments. Coping with information imperfection in natural risk decision process cannot be avoided. Accordingly, an integrated approach (fig. 2) that incorporates uncertainty analysis in the maintenance decision process of deteriorating check dams should be developed.

\subsection{Check dam's deterioration and maintenance Model}

A holistic approach that supports check dam's maintenance decision-making while considering its time-dependent behavior during its lifetime was recently proposed by (Chahrour et al., 2021). The approach consisted in coupling two main models (fig. 2, (a)):

It started by a physics-based model concerning torrential hydraulics and check dam's stability. In this model, the evolution of the torrent's bed when subjected to randomly generated flood series scenarios was analyzed. This analysis was carried out using a 1-D hydraulic software (LOGICHAR). The dimensions of local scouring (erosion) under the dams' foundations were then estimated after each flood event involved in each scenario. After, the time-dependent evolution of normalized degradation indicators related to the external stability of the dam (exceedance of bearing capacity, overturning, and sliding) were assessed considering the following equations:

$$
\mathrm{S}_{B C}=\frac{\sigma_{a d m}-q_{r e f}^{\prime}}{\sigma_{a d m}} \quad S_{O T}=\frac{M_{S}-M_{O}}{M_{S}} \quad S_{S L}=\frac{R_{S L}-R_{H}}{R_{S L}}
$$

where $S_{B C}$ is the bearing capacity stability ratio, $\sigma_{a d m}$ is the soil permissible stress, $q_{r e f}^{\prime}$ is the soil reference stress, $S_{O T}$ is the overturning stability ratio, $M_{S}$ is the stabilizing moment, $M_{O}$ is the overturning moment, $S_{S L}$ is the sliding stability ratio, $R_{H}$ is the resultant horizontal force and $R_{S L}$ is the resisting friction force that opposes sliding.

These indicators were estimated considering possible interactions that exist between local scouring and the external stability of the dam. Finally, a normalized non-dimensional global stability indicator $(\mathrm{Sg})$ combining the three mentioned external stability indicators was defined (Eq. 2) and its evolution was modeled over time. $S g$ belongs to the interval $[0,1]$ where 0 corresponds to a failed state and 1 corresponds to a state with maximal stability level (initial new state). The end purpose of this model was to provide trajectories that enable tracking the dams' deterioration over their lifetime.

$$
S g=\sqrt[3]{S_{B C} \cdot S_{O T} \cdot S_{S L}}
$$


The second model was dedicated for stochastic deterioration and maintenance modeling using physics-informed stochastic Petri nets (SPNs). Four different states of the dams (good, poor, very poor and failed) were defined based on specified thresholds of $\mathrm{Sg}$. Several flood scenarios were generated and modeled using the physics-based model in order to have several time-dependent trajectories of $\mathrm{Sg}$ and thus to have several estimates of transition times between the defined states of the dam. Using these stochastic estimates, empirical non-parametric probability laws corresponding transition times were estimated. These laws were finally used in an SPN model that integrates degradation, inspection, and maintenance processes. Finally, Monte-Carlo simulation has provided stochastic outputs related to (i) the behaviour of the dam when subjected to preventive and/or corrective maintenance operations (time spent by the dam in each state) and (ii) the number of maintenance operations (minor, major, and corrective) carried out over the specified period of simulation. In the developed model, four maintenance strategies were proposed:

- All maintenance operations are allowed.

- Minor preventive operations are inhibited.

- Major preventive operations are inhibited.

- Only corrective operations are allowed.

The obtained results allowed assessing (compare, sort...) several maintenance strategies taking into account the total cost of each strategy. Yet, due to the partial knowledge and imprecise information, input parameters remain imperfect. In other words, different choices for the same input variable may lead to different outputs, and thus influence the decision. Hence, results must be analyzed with caution considering the subjectivity or the quality of the information provided. It is also worthwhile to quantify and propagate the uncertainty through the deterioration and maintenance model so as to gain further information on the confidence that the decision-maker can have in the model outputs.

\subsection{HYRISK: Hybrid approach addressing uncertainty in risk context}

HYRISK is a R package dedicated to handle uncertainty in risk assessments. This package uses hybrid representations of uncertainty, providing functions that jointly handle aleatory (represented by probabilities) and epistemic (represented by possibility distributions, intervals, fuzzy numbers or parametric probability categories, p-boxes) uncertainty. Therefore, HYRISK fills the gap previously identified by treating, altogether, different types of imperfection.

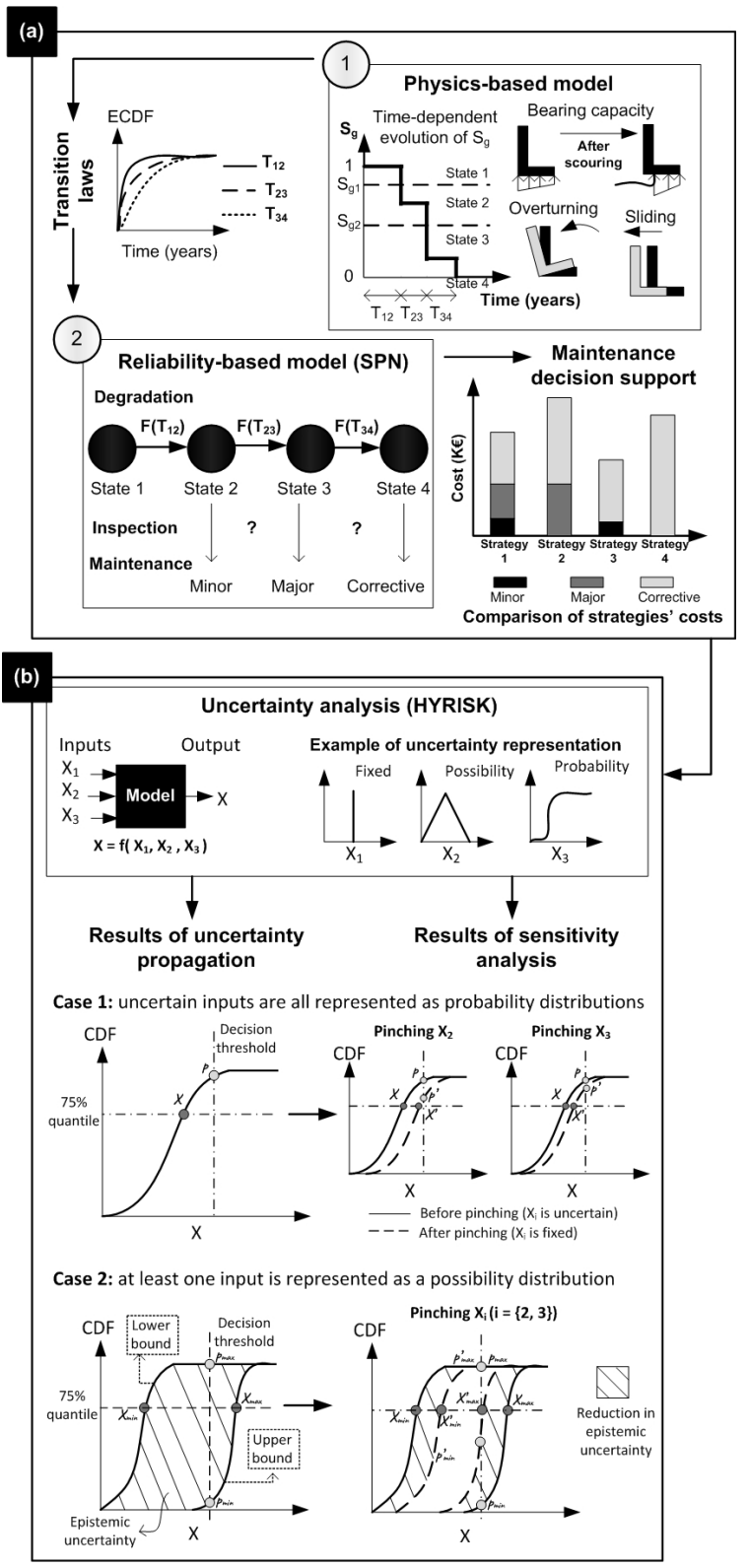

Fig. 2. Integrated methodological framework. (a) Check dam's deterioration and maintenance coupled model; (b) Uncertainty analysis using HYRISK.

\subsubsection{Functionality of HYRISK}

HYRISK package provides functions at each step of the uncertainty quantification process (Rohmer et al. 2018). These steps and the associated functions are summarized below.

(1) Uncertainty representation: In this step, input variables (imprecise, random or fixed) are first defined using CREATE_INPUT function. 
Then, the CREATE_DISTR function is used to assign probability (normal, uniform, Gumbel, etc...) for random input variables and possibility distributions (trapezoidal or triangular) for imprecise ones. The graphical representation forms of all input parameters can be finally be implemented using PLOT_INPUT function.

(2) Uncertainty propagation: The PROPAG function evaluates impact of the uncertainty that invades the inputs on the outcome of the risk assessment model. It combines random sampling using Monte Carlo method with fuzzy interval analysis for jointly handling possibility, probability distributions and p-boxes.

(3) Sensitivity analysis: This step is dedicated for analyzing how uncertainty in the model's output is influenced by each model's input separately. The pinching method, implemented using PINCHING_fun and SENSI_PINCHING functions is used in this analysis.

\subsubsection{Results produced with HYRISK}

Both uncertainty propagation and sensitivity analysis via HYRISK result in a pair of upper and lower cumulative probability distributions (CDFs), which defines the p-box related to the uncertainty on the output parameter X (fig. 2, (b)). Three statistical quantities that support the decision-making process can be extracted from the resulted p-boxes using the following HYRISK functions:

- PROBAIINTERVAL provides the upper and lower bound on the probability of $\mathrm{X}$ being below a decision threshold.

- QUAN_INTERVAL provides the upper and lower bound corresponding to a quantile at a given level (e.g. 75\%).

- UNCERTAINTY estimates the area within the lower and upper probability distribution thus providing a global measure of epistemic uncertainty.

\section{Application: Uncertainty Propagation Within the Check Dam's Model}

The analysis of information imperfection should be carried out considering its propagation through the different stages of the check dam's model. Indeed, the integrated model can be divided into sub-models (fig. 3) at which uncertainty analysis can be implemented. In this paper, a simple application is performed, in which HYRISK functions are executed on a sub-model of the physics-based model related to local scouring estimation.

\subsection{Uncertainty representation}

The phenomenon of scouring corresponds to soil erosion downstream and under a structure's foundation. The dimensions of the generated scour pit

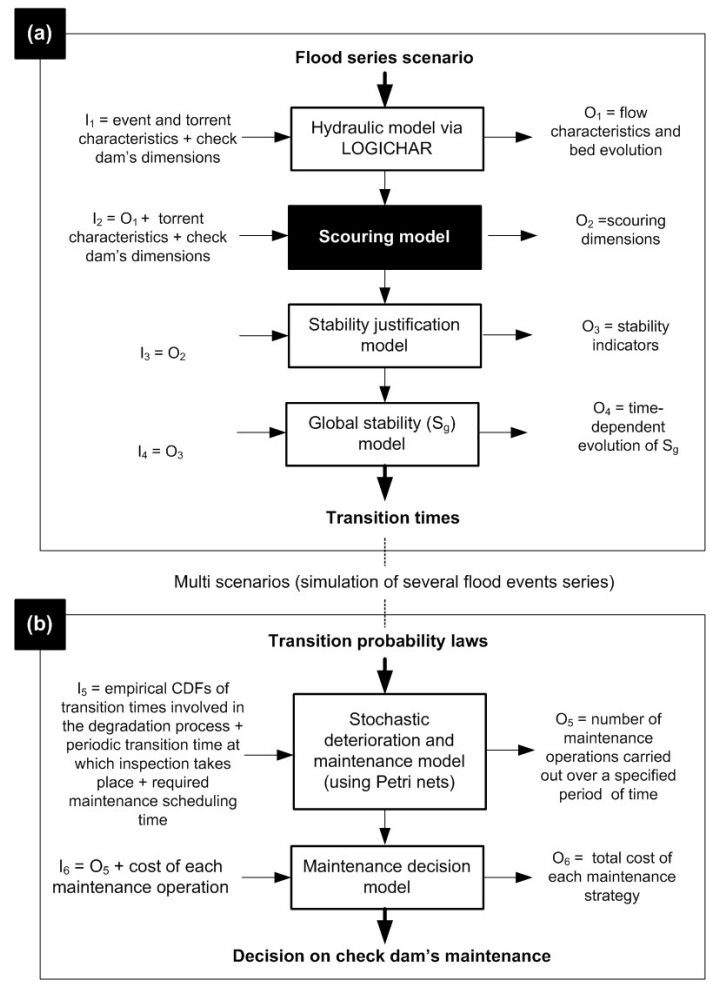

Fig. 3. Sub-models involved in the (a) physics-based model and the (b) reliability-based model of a check dam. $I_{i}$ : list of inputs to sub-model $i . O_{i}$ : list of outputs provided by sub-model $i$.

depend on specific characteristics related to the flow, the torrent and the dam. The main dimensions that have an influence on the external stability of the dam are the scouring depth $\left(S_{d}\right)$ and the scouring width $\left(S_{w}\right)$ illustrated in fig. 4 . Based on the scouring approach developed by (Chahrour et al. 2021), the estimation of $S_{d}$ and $S_{w}$ requires the following inputs:

$$
\begin{gathered}
S_{d}=f\left(H_{s}, h_{s}, B, D_{90}, Z_{F i}, d_{z}, \Delta, H_{w}, b, \theta\right) \\
S_{w}=f\left(H_{s}, h_{s}, B, D_{90}, Z_{F i}, d_{z}, \Delta, H_{w}, b\right)
\end{gathered}
$$

where $H_{s}$ is the hydraulic head, $h_{s}$ is the downstream water level, $B$ is the torrent's channel width, $D_{90}$ is the grain size for which $90 \%$ of the sediment in weight is finer, $Z_{F i}$ is the initial bed level downstream the dam, $d_{z}$ is the drop in bed level, $\Delta$ is the sediment relative submerged density, $H_{w}$ is the dam's height below the weir's base, $b$ is width of the weir's base and $\theta$ is the downstream slope of the scour pit under the foundation.

In the present application, $D_{90}, Z_{F i}$ and $\theta$ are 


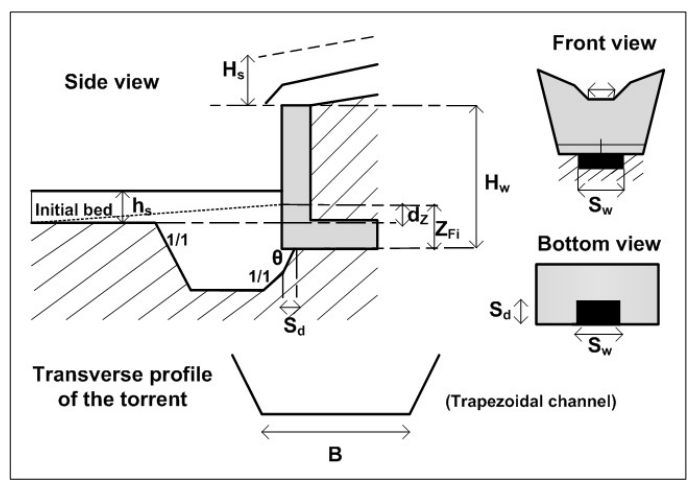

Fig. 4. Local scouring dimensions under a check dam's foundation.

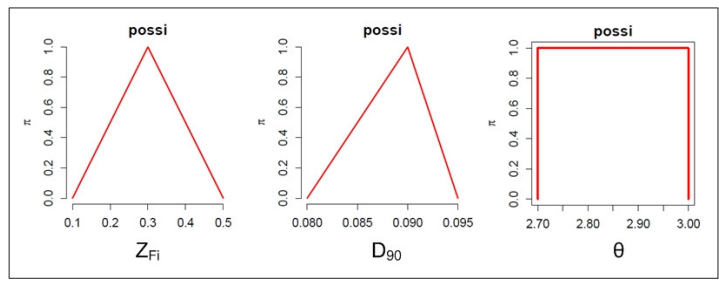

Fig. 5. Representation of uncertain inputs. $Z_{F i}(m), D_{90}(m)$ : possibility distribution (triangular) and $\theta(\mathrm{m} / \mathrm{m})$ possibility distribution (interval).

considered as epistemic uncertain parameters following a possibility distribution (fig. 5). All other parameters are fixed to values given in table 1 .

Table 1. Fixed inputs used for scouring calculation.

\begin{tabular}{llllllll}
\hline Input & $\begin{array}{l}H_{s} \\
(\mathrm{~m})\end{array}$ & $\begin{array}{l}h_{s} \\
(\mathrm{~m})\end{array}$ & $\begin{array}{l}B \\
(\mathrm{~m})\end{array}$ & $\begin{array}{l}d_{z} \\
(\mathrm{~m})\end{array}$ & $\Delta$ & $\begin{array}{l}H_{w} \\
(\mathrm{~m})\end{array}$ & $\begin{array}{l}b \\
(\mathrm{~m})\end{array}$ \\
\hline Value & 0.737 & 0.229 & 6 & 0.873 & 1.65 & 2.3 & 4.4 \\
\hline
\end{tabular}

\subsection{Uncertainty propagation and sensitivity analysis}

Uncertainty analysis is performed in order to assesses the uncertainty associated with estimating the dimensions of scouring under the dam's foundation. To do this, the uncertainty represented in fig. 5 is propagated with the IRS algorithm of (Baudrit et al., 2007) and 1000 random samples. Such propagation results in a pair of upper and lower cumulative probability distributions (CDFs) for each of $S_{d}$ and $S_{w}$, presented in fig. 6. The area bounded between the lower and the upper curves represents the epistemic uncertainty.

Due to the fact that there exist more than one imprecise or missing parameter, a sensitivity anal-

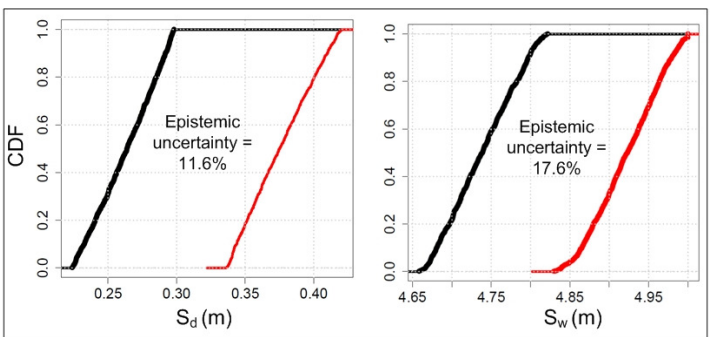

Fig. 6. Results of the hybrid uncertainty propagation representing the lower (black curve) and upper (red curve) CDFs related to the uncertainty on $S_{d}$ and $S_{w}$.

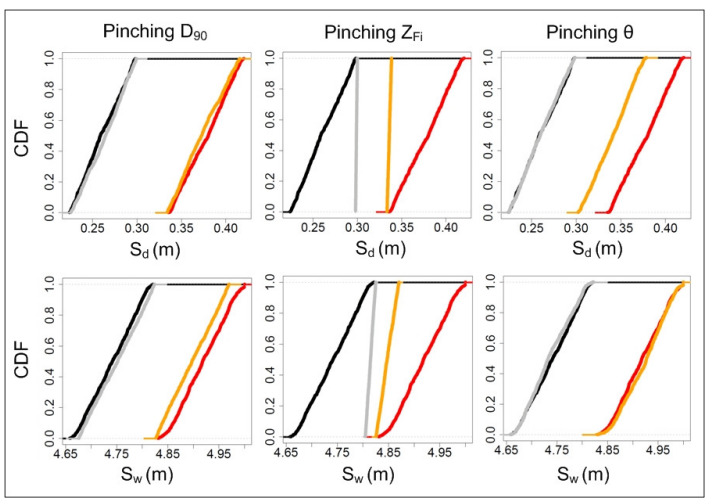

Fig. 7. Lower and upper CDFs related to the uncertainty on $S_{d}$ and $S_{w}$ before (black and red curves) and after (grey and orange curves) pinching the parameters $D_{90}, Z_{F i}$ and $\theta$ respectively to the values of $0.09 \mathrm{~m}$, $0.3 \mathrm{~m}$ and $3 \mathrm{~m} / \mathrm{m}$.

ysis based on pinching approach is carried out. It consists of assessing how the imprecision on $S_{d}$ and $S_{w}$ may be reduced if additional information on the input parameters $D_{90}, Z_{F i}$ and $\theta$ were available. In the present application, the pinching function is used in order to transform the triangular possibility distributions of $D_{90}$ and $Z_{F i}$ and the interval associated to the parameter $\theta$ into fixed values of: $0.09 \mathrm{~m}, 0.3 \mathrm{~m}$ and $3 \mathrm{~m} / \mathrm{m}$. Figure 7 shows the evolution of the lower and upper CDFs corresponding to $S_{d}$ and $S_{w}$ after pinching $D_{90}$, $Z_{F i}$ and $\theta$.

The impact of pinching $D_{90}, Z_{F i}$ and $\theta$ on reducing the epistemic uncertainty (quantity of interest) can be summarized in terms of the reduction of the area between both the lower and the upper CDFs. Results, provided in table 2 reveal that $S_{d}$ and $S_{w}$ are more sensitive to the epistemic parameter $Z_{F i}$. It can be also seen that $D_{90}$ does not have a great influence on $S_{d}$. Note that $S_{w}$ is not sensitive to the parameter $\theta$ since it is independent of it. In terms of decisions, such sensitivity analysis, provides information to risk 
Table 2. Epistemic uncertainty (\%) and its percentage reduction between the one obtained from the uncertainty propagation and that obtained after pinching input parameters.

\begin{tabular}{ccccc}
\hline \multirow{2}{*}{ Output } & Uncertainty & \multicolumn{4}{c}{ Pinched input parameter } \\
& & $D_{90}$ & $Z_{F i}$ & $\theta$ \\
\hline \multirow{2}{*}{$S_{d}$} & \multirow{2}{*}{11.60} & 10.84 & 3.67 & 7.93 \\
& & $6.55 \%$ & $68.36 \%$ & $31.63 \%$ \\
\hline \multirow{2}{*}{$S_{W}$} & \multirow{2}{*}{17.60} & 14.56 & 3.36 & 17.60 \\
& & $17.27 \%$ & $80.90 \%$ & $0 \%$ \\
\hline
\end{tabular}

managers and decision-makers concerning critical indicators that should be given deep attention. For example, in this case, as it is revealed that $Z_{F i}$ has a big influence on $S_{d}$, countermeasures and maintenance decisions must consider limiting the evolution of the bed level behind the dam (e.g. adding earth or concrete riprap).

\section{Conclusion}

The propagation of information imperfection within a check dam's deterioration and maintenance model and its effect on the final decision cannot be disregarded. The functional design and structural justification of check dams are known but are based on hypotheses that are not always formalized or available. Indeed, topographic, geotechnical and hydraulic data may be missing or imprecise. When confronted with epistemic uncertainty, conventional probability theory tools frequently appear too restrictive. The HYRISK package propagates and uses random and epistemic uncertainties together thus providing a hybrid approach sufficient for dealing with different forms of imperfection. At a practical level, it combines possibilistic and probabilistic approaches, thus considering that an imprecise or unknown variable is described by a framing of an upper and lower distributions instead of a single probability distribution. In this paper, uncertainty analysis was performed only on the scouring sub-model in order to describe technically the functionalities of HYRISK as a first stage. Further analysis will be carried out on the different sub-models involved within the deterioration and maintenance model of a torrent check dam so as to investigate how the modeled uncertainty on the dam stability can be integrated into the maintenance model, and how it eventually affects the maintenance decision.

\section{Acknowledgement}

This work is supported by the French National Research Agency in the framework of the Investissements
d'Avenir program (ANR-15-IDEX-02) with a contribution of the French Ministry for Ecological and Solidary Transition (MTES/DGPR) in charge of risk prevention. In addition, thanks to Aurelien Beuerle (Master 1 Grenoble INP ENSE3), who has contributed during his master's internship in this research work.

\section{References}

Baudrit, C., D. Guyonnet, and D. Dubois (2007, 09). Joint propagation of variability and imprecision in assessing the risk of groundwater contamination. Journal of contaminant hydrology 93, 72-84.

Carladous, S., J.-M. Tacnet, M. Batton-Hubert, J. Dezert, and O. Marco (2019). Managing protection in torrential mountain watersheds: A new conceptual integrated decision-aiding framework. Land Use Policy 80, $464-479$.

Chahrour, N., M. Nasr, J.-M. Tacnet, and C. Bérenguer (2021). Deterioration modeling and maintenance assessment using physics-informed stochastic petri nets: Application to torrent protection structures. Reliability Engineering \& System Safety 210, 107524.

Chojnacki, E., J. Baccou, and S. Destercke (2010). Numerical accuracy and efficiency in the propagation of epistemic and aleatory uncertainties. International Journal of General Systems 39(7), 683-704.

Dubois, D et Prade, H. (1989). Possibility Theory: an approach to computerized processing of uncertainty. Plenum Press, New York.

Helton, J. and W. Oberkampf (2004, 07). Alternative representations of epistemic uncertainty. Reliability Engineering \& System Safety 85, 1-10.

Rohmer, J., J.-C. Manceau, D. Guyonnet, F. Boulahya, and D. Dubois (2018, April). An R package for hybrid uncertainty analysis in natural and environmental risk assessments using probability, imprecise probability and possibility distributions. In $E G U$ 2018 - European Geosciences Union General Assembly 2018, Vienne, France.

Shafer, G. (1976). A Mathematical Theory of Evidence. Princeton University Press.

Tacnet, J.-M., J. Dezert, C. Curt, M. Batton-Hubert, and E. Chojnacki $(2014,06)$. How to manage natural risks in mountain areas in a context of imperfect information? New frameworks and paradigms for expert assessments and decision-making. Environment Systems and Decisions 34, 288--311.

Tacnet, J.-M. and D. Richard (2010). De la conception à la sûrete des barrages de correction torrentielle. Sciences Eaux \& Territoires, (2);164-175.

Walley, P. (1991). Statistical Reasoning with Imprecise Probabilities. Chapman \& Hall.

Zadeh, L. (1965). Fuzzy sets. Information and Control 8(3), 338-353. 